\title{
A Case of Iris Melanocytoma Demonstrating Diffuse Melanocytic Proliferation with Uncontrolled Intraocular Pressure
}

\author{
Mami Kusunose $^{a}$ Yuji Sakino ${ }^{a}$ Yoshihiro Noda ${ }^{a}$ Tsutomu Daa ${ }^{b}$ \\ Toshiaki Kubota ${ }^{a}$ \\ ${ }^{a}$ Department of Ophthalmology, Oita University Faculty of Medicine, Yufu, Japan; \\ ${ }^{b}$ Department of Pathology, Oita University Faculty of Medicine, Yufu, Japan
}

\section{Keywords}

Melanocytoma $\cdot$ Secondary glaucoma $\cdot$ Trabecular meshwork $\cdot$ Iris · Ciliary body

\begin{abstract}
We report a rare case with histologically proven melanocytoma of the iris that demonstrated diffuse melanocytic proliferation with uncontrolled secondary glaucoma and investigate the etiology of the intraocular pressure elevation. The patient was a 78-year-old man with a history of darkened iris of his left eye. The intraocular pressure was $39 \mathrm{~mm} \mathrm{Hg}$. A slit-lamp examination showed a diffuse darkened iris, and a gonioscopic examination revealed open angle with circumferential heavy pigmentation. There was no pigment dispersion of the anterior chamber and no pigment deposition of the cornea. We suspected malignant ring melanoma in the left eye and enucleated it. The globe was examined with light and electron microscopy. Light microscopy revealed the presence of heavily pigmented tumor cells in the iris, ciliary body, trabecular meshwork, and Schlemm's canal. A bleached preparation showed large tumor cells with central and paracentral nuclei without mitosis. Electron microscopy of the trabecular meshwork revealed melanin-bearing tumor cells invading the intertrabecular spaces, and the melanin granules were not phagocytosed in the trabecular cells. The mechanical obstruction of the aqueous flow by the tumor cells may be a major cause of secondary glaucoma in eyes with iris melanocytoma presenting diffuse proliferation.
\end{abstract}




\section{Case Reports in Ophthalmology}

\section{Introduction}

Melanocytoma presents as a deeply pigmented tumor and rarely arises in the iris or ciliary body of the eye. It usually appears as a focal tumor, slowly enlarging and invading the anterior chamber angle, and may develop secondary glaucoma $[1,2]$. The mechanism of the intraocular pressure elevation has not yet been elucidated. We herein report a patient with histologically proven melanocytoma of the iris who demonstrated diffuse melanocytic proliferation with uncontrolled secondary glaucoma and investigate the etiology of the intraocular pressure elevation with electron microscopy.

\section{Case Report}

A 78-year-old man visited us with a history of darkened iris of his left eye. His visual acuity was 0.4 in the right eye and "hand motion" in the left. His intraocular pressure was 15 $\mathrm{mm} \mathrm{Hg}$ in the right eye and $39 \mathrm{~mm} \mathrm{Hg}$ with medication in the left. A slit-lamp examination of the left eye showed a diffuse darkened iris, and a gonioscopic examination revealed open angle with heavy circumferential pigmentation. There was no pigment dispersion of the anterior chamber and no pigment deposition of the cornea (Fig. 1). Optic disc and perimetry showed advanced glaucomatous changes in the left eye. A fundus examination showed diffuse chorioretinal atrophy of unknown etiology in both eyes. An ultrasound biomicroscopic examination revealed thickening of the ciliary body at the temporal position in the left eye. A PET-CT examination showed no abnormal findings in other organs. The patient had lost light perception of the left eye in 1 month. We suspected malignant ring melanoma in the left eye and enucleated it after discussing our suspicions with the patient.

The globe was fixed in $10 \%$ neutral buffered formalin. A gross examination of the cut section of the globe showed a deeply pigmented lesion involving the iris and ciliary body. A piece of the anterior segment was fixed in a solution of $4 \%$ glutaraldehyde with $0.1 \mathrm{M}$ cacodylate buffer for $24 \mathrm{~h}$, postfixed with $1.0 \% \mathrm{OsO}_{4}$, dehydrated in graded ethanol, and then embedded in an epoxy resin. Ultrathin sections were stained with uranyl acetate and lead citrate and then examined by electron microscopy. Hematoxylin eosin staining of a paraffin section of the anterior segment revealed the presence of heavily pigmented tumor cells in the iris, ciliary body, trabecular meshwork and Schlemm's canal. These tumor cells had invaded the trabecular meshwork and Schlemm's canal. A bleached preparation revealed large tumor cells with central and paracentral nuclei. The tumor cells showed a low nuclear-tocytoplasmic ratio with small or inconspicuous nucleoli. Mitosis was absent. Electron microscopy of the trabecular meshwork revealed melanin-bearing tumor cells invading the intertrabecular spaces, and the melanin granules were not phagocytosed in the trabecular cells (Fig. 2).

\section{Discussion}

Iris melanocytoma typically presents as a dark-pigmented iris nodule with a mossy, irregular surface $[1,2]$, while iris ring melanoma is a rare variant of uveal melanoma that manifests as a circumferential, flat tumor growth predominantly confined to the anterior chamber angle structure [3]. We experienced a very rare case of diffuse melanocyticinvading melanocytoma mimicking iris ring melanoma [4]. The clinical and histological find- 
ings showed no pigment dispersion from melanocytoma cells in this case. The present electron microscopy findings reveal that the mechanical obstruction of the aqueous flow by the tumor cells was a major cause of secondary glaucoma, as the melanin granules were not phagocytosed by the trabecular cells, which is observed in glaucomatous eyes with pigment dispersion syndrome.

Although the beginning of diffuse darkening of the iris of the affected eye is obvious, the anterior segment of the right eye shows a normal appearance. The choroidal nevus carries a small risk of malignant transformation [5]; however, in our search of the literature, we found no reports on uveal melanoma or melanocytoma associated with chorioretinal atrophy. We conclude that iris melanocytoma occurred by chance in this patient with diffuse chorioretinal atrophy in both eyes.

\section{Acknowledgements}

The authors would like to thank Dr. Hideto Yatsuka, Yatsuka Eye Clinic, Saeki, Japan, who referred the patient.

\section{Statement of Ethics}

The patient has consented to the submission of this case report to the journal.

\section{Disclosure Statement}

None of the authors has any proprietary interests or conflicts of interest related to this submission. None of the authors received any financial support.

\section{References}

1 LoRusso FJ, Boniuk M, Font RL: Melanocytoma (magnocellular nevus) of the ciliary body: report of 10 cases and review of the literature. Ophthalmology 2000;107:795-800.

-2 Demirci H, Mashayekhi A, Shields CL, Eagle RC, Shields JA: Iris melanocytoma: clinical features and natural course in 47 cases. Am J Ophthalmol 2005;139:468-475.

-3 Demirci H, Shields CL, Shields JA, Eagle RC, Honavar S: Ring melanoma of the anterior chamber angle: a report of fourteen cases. Am J Ophthalmol 2001;132:336-342.

4 Biswas J, D'Souza C, Shanmugam MP: Diffuse melanotic lesion of the iris as a presenting feature of ciliary body melanocytoma: report of a case and review of the literature. Surv Opthalmol 1998;42:378382.

5 Chien JL, Sioufi K, Surakiatchanukul T, Shields JA, Shields CL: Choroidal nevus: a review of prevalence, features, genetics, risks, and outcomes. Curr Opin Ophthalmol 2017, Epub ahead of print. 


\section{Case Reports in Ophthalmology}
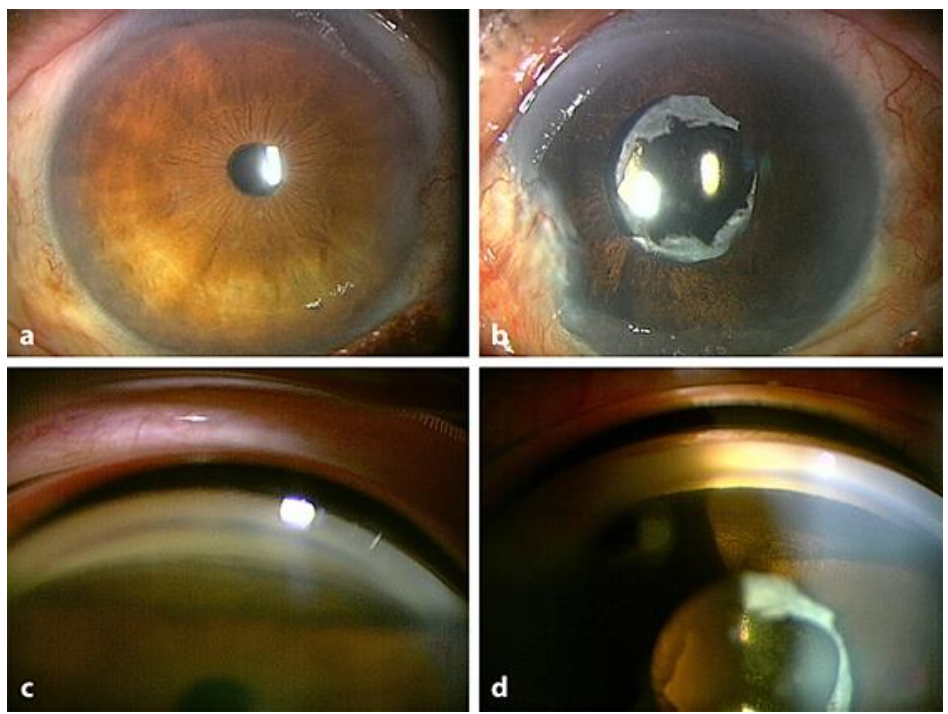

Fig. 1. a Slit-lamp photograph showing the normal appearance of the iris in the right eye. b Diffuse brown pigmentation of the iris in the left eye is presented. c Gonioscopy of the right eye revealing a normal open angle. $\mathbf{d}$ Gonioscopy of the left eye showing an open angle with circumferential heavy pigmentation.

Kusunose et al.: A Case of Iris Melanocytoma Demonstrating Diffuse Melanocytic Proliferation with Uncontrolled Intraocular Pressure 


\section{Case Reports in Ophthalmology}

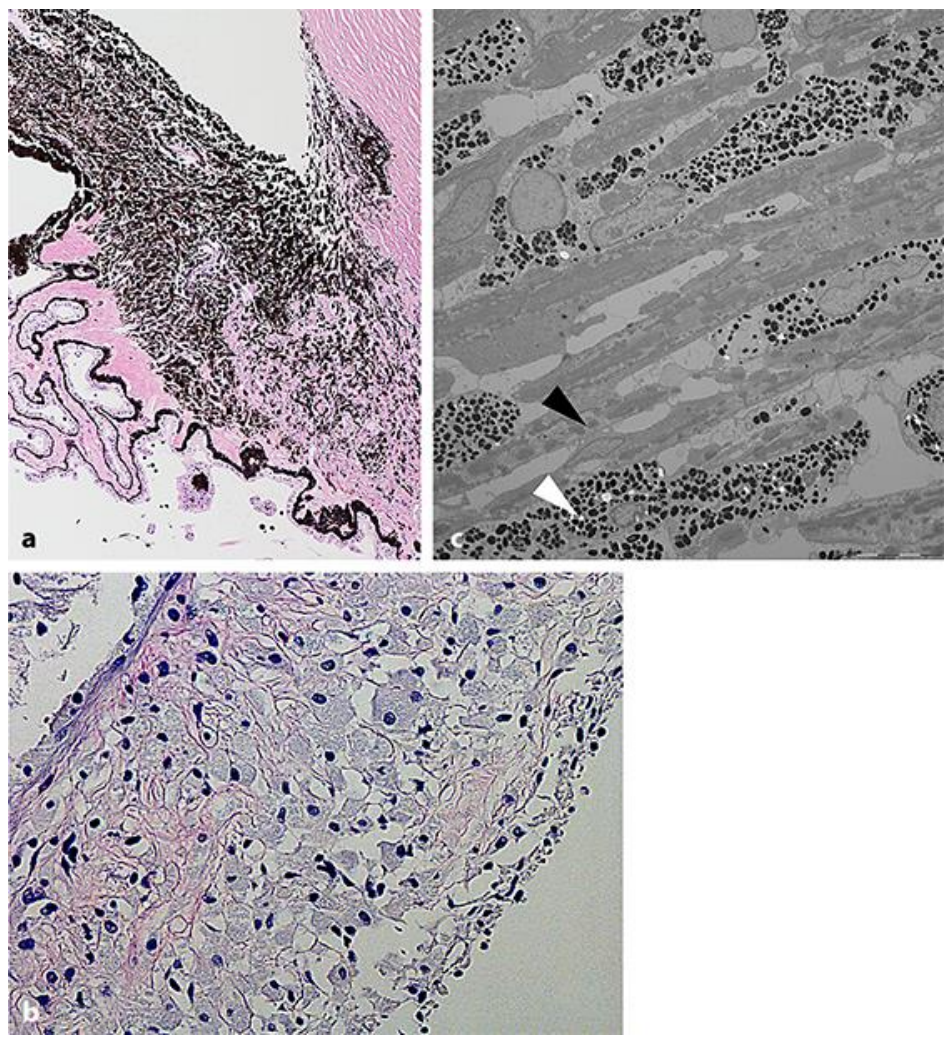

Fig. 2. a Histological microphotograph showing a densely pigmented mass involving the iris, ciliary body, trabecular meshwork, and Schlemm's canal. Hematoxylin and eosin. 40×. b A bleached preparation revealing large tumor cells with central and paracentral nuclei. The tumor cells show a low nuclear-tocytoplasmic ratio without mitosis (bleached preparation. Hematoxylin and eosin. 200x). c An ultrastructural microphotograph showing melanin-containing tumor cells (white arrow) and trabecular meshwork cells (black arrow) in the trabecular meshwork. The melanin granules are not phagocytosed by the trabecular cells (electron micrograph, 2,000x). 